\title{
Mitigating Attacks on Open Functionality in SMS-Capable Cellular Networks
}

\author{
Patrick Traynor, William Enck, Patrick McDaniel, and Thomas La Porta \\ Systems and Internet Infrastructure Security Laboratory \\ Networking and Security Research Center \\ Department of Computer Science and Engineering \\ The Pennsylvania State University \\ University Park, PA 16802 \\ \{traynor, enck, mcdaniel, tlp\}@cse.psu.edu
}

\begin{abstract}
The transformation of telecommunications networks from homogeneous closed systems providing only voice services to Internetconnected open networks that provide voice and data services presents significant security challenges. For example, recent research illustrated that a carefully crafted DoS attack via text messaging could incapacitate all voice communications in a metropolitan area with little more than a cable modem. This attack highlights a growing threat to these systems; namely, cellular networks are increasingly exposed to adversaries both in and outside the network. In this paper, we use a combination of modeling and simulation to demonstrate the feasibility of targeted text messaging attacks. Under realistic network conditions, we show that adversaries can achieve blocking rates of more than $70 \%$ with only limited resources. We then develop and characterize five techniques from within two broad classes of countermeasures - queue management and resource provisioning. Our analysis demonstrates that these techniques can eliminate or extensively mitigate even the most intense targeted text messaging attacks. We conclude by considering the tradeoffs inherent to the application of these techniques in current and next generation telecommunications networks.
\end{abstract}

\section{Categories and Subject Descriptors}

C.2.0 [Computers-Communication Networks]: General-Security and protection

\section{General Terms}

Security

\section{Keywords}

telecommunications, sms, denial-of-service, open-functionality

\section{INTRODUCTION}

In addition to traditional voice communications, cellular systems offer a wide variety of data and text/short messaging services

Permission to make digital or hard copies of all or part of this work for personal or classroom use is granted without fee provided that copies are not made or distributed for profit or commercial advantage and that copies bear this notice and the full citation on the first page. To copy otherwise, to republish, to post on servers or to redistribute to lists, requires prior specific permission and/or a fee.

MobiCom'06, September 23-26, 2006, Los Angeles, California, USA.

Copyright 2006 ACM 1-59593-286-0/06/0009 ...\$5.00.
(SMS). Cellular providers have introduced SMS gateways between the phone networks and the Internet to increase the reach (and volume) of text messaging. These gateways are partially responsible for the soaring usage of text messaging. Some five billion text messages are sent each month in the United States alone [21]. Indeed, for significant numbers of users, text messaging has become the primary means of communication [2].

Such interconnectivity is not without serious security risks. The cellular infrastructure was designed to operate as a homogeneous and highly controlled system. Interconnectivity with the Internet invalidates many of the assumptions upon which the phone networks were designed. These failing assumptions lead to critical vulnerabilities. Enck et al. [12] showed that, under a simple model, small but carefully crafted volumes of text messages could be used to incapacitate all cellular communications in large metropolitan areas. Particularly in times of crisis, the cost of the maliciouslydriven loss of cellular communications could be immeasurable.

In this paper, we consider both the reality and mitigation of previously postulated attacks on text messaging. Using analytical modeling and highly detailed simulation of the GSM air interface, we show that the simple model described in the original study underestimated the traffic volumes necessary to to effect a high-impact attack by approximately a factor of three. However, while the specific estimates made by Enck et al. were not perfect, we found their qualitative arguments of feasibility to hold under a range of realistic traffic models. In short, our findings demonstrate that cellular networks are in fact quite vulnerable to SMS-based attacks mounted by adversaries with even limited resources. Specifically, an attack capable of preventing the large majority of voice communications in a metropolitan area is indeed possible with the bandwidth available to a single cable modem.

In the presence of this reality, we have developed five techniques from within two broad classes of countermeasures, queue management and resource provisioning, to combat these attacks. Our goal is to insulate voice call requests and the delivery of high priority text messages from the attack. We apply well-known queuing techniques including variants of Weighted Fair Queuing (WFQ), and Weighted Random Early Detection (WRED), which are well tested for addressing traffic overload in the Internet. These schemes attempt to provide differentiated service to voice and data, and hence alleviate resource contention.

The second class of solutions reapportion the wireless medium using the novel Strict Resource Provisioning (SRP), Dynamic Resource Provisioning (DRP) and Direct Channel Allocation (DCA) algorithms. Our modeling and simulation analyses of the countermeasures demonstrates their utility: the effect of the solutions 
ranged from partial attack mitigation for both flows to total elimination of attack-related voice blocking and the successful delivery of high priority text messages. A further exploration of the deployment of these solutions highlights a number of security, performance, and complexity tradeoffs. We discuss these tradeoffs throughout and make a number of recommendations to the community.

In this work, we make the following contributions:

- Network/Attack Characterization: We create a realistic characterization of system behavior under targeted SMS attacks. Such characterizations dually ascertain their realism and develop a profile of the effect of an attack under varying traffic intensity and arrival models.

- Current Countermeasure Analysis: We briefly consider popularly advertised solutions to targeted text messaging attacks. Principally, we find that the currently deployed "edge solutions" are largely ineffective against all but the most naïve attack.

- Countermeasure Development and Evaluation: We develop and characterize a number of countermeasures adapted from well-established queuing techniques and novel channel allocation strategies. Our analysis demonstrates that these attacks can be effectively mitigated by altering the traffic handling disciplines at the air interface. Hence, countering these attacks need not require a substantive change to internal structure or operation of cellular networks, but can be handled entirely by software changes at the base station.

The remainder of this paper is organized as follows: Section 2 discusses related work; Section 3 provides an overview of cellular signaling networks and characterizes targeted SMS attacks; Section 4 offers a number of mitigation strategies and models their ability to mitigate these attacks; Section 5 details the attack and mitigation simulations; and Section 6 offers concluding remarks and future work.

\section{RELATED WORK}

Physical disconnection from external networks has long been one of the most effective means of providing security for telecommunication systems. Accordingly, security in these networks has traditionally centered around the prevention of fraudulent access and billing. The changing needs of users, however, have forced the gradual erosion of well defined network borders. Whether due to new access patterns or the advent of new services (e.g. data networking via the Internet), systems that once relied upon isolation as a major portion of their defenses are no longer able to do so. Because fundamental assumptions about the underlying architecture of the critical communications infrastructure have changed, security measures addressing new classes of threats resulting from the interconnection of networks are essential. Similar observations and concerns have been expressed in the National Strategy to Secure Cyberspace [1].

Telecommunications networks are not the only systems to suffer from vulnerabilities related to expanded connectivity. Systems including Bank of America's ATMs and 911 emergency services for Bellevue, Washington were both made inaccessible by the Slammer worm [24]. Although neither system was the target of this attack, simply being connected to the Internet made them experience significant collateral damage. Systems less directly connected to the Internet have also been subject to attack. Byers, et al. [8] demonstrated one such attack using simple automated scripts and webforms. Immense volumes of junk postal mail could then be used to launch Denial of Service (DoS) attacks on individuals.

The typical targets of DoS attacks, however, are more traditional online resources. In 2000, for example, users were unable to reach Amazon, eBay and Yahoo! as their servers were bombarded with over a gigabit per second of traffic [31]. Since that time, sites ranging from software vendors [14] and news services [33] to online casinos [6] have all fallen victim to such attacks. While significant research has been dedicated to categorizing [23], mitigating [34, 16] and eliminating [37] these attacks, no solutions have seen widespread implementation. Because of the various transformations of data transiting between the Internet and telecommunications networks, the direct application of the above techniques would be ineffective.

Whether accidental or the result of malicious behavior, denial of service incidents have been studied and documented in telecommunications networks. The National Communications System published a study on the effects of text messages during emergency situations. Given realistic scenarios for usage, this technical bulletin argued that SMS resources needed to be increased 100-fold in order to operate under such conditions [27]. Operators have also reported problems with connectivity during holidays due to increased volumes of SMS traffic [22]. Enck, et al. [12] demonstrated that an adversary would be able to cause the same congestion in targeted metropolitan areas by injecting a relatively small amount of traffic. While a number of solutions were proposed in that work, none have yet been measured and compared.

\section{SYSTEM/ATTACK CHARACTERIZATION}

\subsection{Message Delivery Overview}

In the following subsection, we provide a high-level, simplified tutorial on text message delivery in cellular networks.

\subsubsection{Message Insertion}

Messages may be submitted into the system from cell phones operating within the system, via web portals managed by the service providers, or from external sources such as email, etc. We focus on delivery from external sources, but the message flow for all types of messages is similar.

An Internet-originated SMS message can be generated by any one of a number of External Short Messaging Entities (ESMEs). ESMEs include devices and interfaces ranging from email and webbased messaging portals to service provider websites and voice mail services and can be attached to telecommunications networks either by dedicated connection or the Internet. When a message is injected into the network, it is delivered to the Short Messaging Service Center (SMSC). These servers are responsible for the execution of a "store-and-forward" protocol that eventually delivers text messages to their intended destination.

The contents and destination information from the message are examined by the SMSC and are then copied into a properly formatted packet. At this point, messages originating in the Internet and those created in the network itself become indistinguishable. Formatted text messages are then placed in an egress queue in the SMSC and await service.

\subsubsection{Message Routing}

Before an SMSC can forward a text message to a targeted mobile device, it must first determine the location of that device. To accomplish this, the SMSC queries a database known as the Home Location Register (HLR). The HLR is responsible for storing subscriber data including availability, billing information, available 


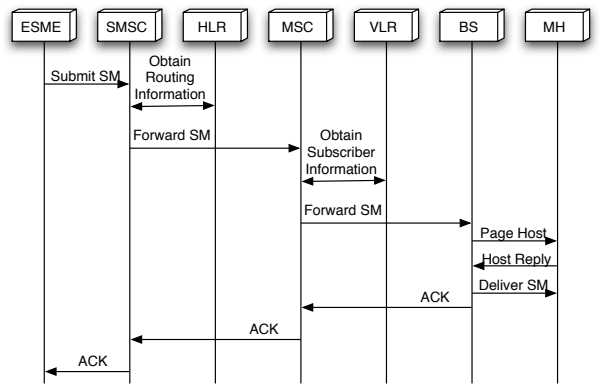

Figure 1: A high level description of SMS delivery in an SS7 network.

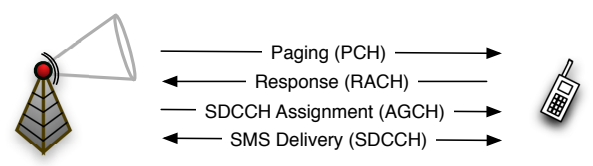

Figure 2: An overview of SMS message delivery on the wireless or air interface. Incoming voice calls would follow a similar procedure except that they would receive a $\mathbf{T C H}$ after using the SDCCH.

services and current location. With the help of other elements in the network, the HLR determines the routing information for the targeted device. If the desired phone is not available, the SMSC stores the message until a later time for subsequent retransmission. Otherwise, the SMSC receives the address of the Mobile Switching Center (MSC) currently providing service to the target device. The MSC delivers the text message over the wireless interface through its attached Base Stations (BS). Figure 1 illustrates the path described above.

\subsubsection{Wireless Delivery}

An area of coverage in a wireless network is called a cell. Each cell is typically partitioned into multiple (usually three) sectors. We characterize the system on a per sector basis throughout the paper.

The air interface, or radio portion of the network, is traditionally divided into two classes of logical channels - the Control Channels (CCHs) and Traffic Channels (TCH). TCHs carry voice traffic after call setup has occurred. $\mathrm{CCHs}$, which transport information about the network and assist in call setup/SMS delivery, are subclassified further. In order to alert a targeted device that a call or text message is available, a message is broadcast on the Paging Channel (PCH). Note that multiple base stations broadcast this page in an attempt to quickly determine the sector in which the targeted recipient is located. Upon hearing its temporary identifier on the $\mathrm{PCH}$, available devices inform the network of their readiness to accept incoming communications using the slotted ALOHA-based Random Access Channel (RACH) uplink. A device is then assigned a Standalone Dedicated Control Channel (SDCCH) by listening to the Access Grant Channel (AGCH). If a text message is available, the base station authenticates the device, enables encryption, and then delivers the contents of the message over the assigned SDCCH. If instead a call is incoming for the device, the SDCCH is used to authenticate the device and negotiate a TCH for voice communications.

Figure 2 offers an overview of the wireless portion of message delivery.

\subsection{System Vulnerability}

All large scale attacks, whether targeting the digital or physical domain, evolve in the following phases: recognition (identification
Table 1: Commonly Used Variables

\begin{tabular}{|c|l|}
\hline$\lambda_{\text {call }}$ & Arrival rate of voice calls \\
\hline$\lambda_{S M S}$ & Arrival rate of text messages \\
\hline$\mu_{S D C C H, \text { call }}$ & Service rate of voice calls at SDCCH \\
\hline$\mu_{T C H, \text { call }}$ & Service rate of voice calls at TCH \\
\hline$\mu_{S D C C H, S M S}$ & Service rate of text messages at SDCCH \\
\hline$\rho_{\text {call }}$ & Call traffic intensity \\
\hline$\rho_{S M S}$ & SMS traffic intensity \\
\hline
\end{tabular}

Table 2: System and Attack Parameters

\begin{tabular}{|l|l|}
\hline$\mu_{T C H}^{-1}$ & $120 \mathrm{sec}[28]$ \\
\hline$\mu_{S D C C H, \text { call }}^{-1}$ & $1.5 \mathrm{sec}[28]$ \\
\hline$\mu_{S D C C H, S M S}^{-1}$ & $4 \mathrm{sec}[27]$ \\
\hline$\lambda_{\text {call }}$ & $50,000 \mathrm{calls} / \mathrm{city} / \mathrm{hr}$ \\
& $.2525 \mathrm{calls} / \mathrm{sector} / \mathrm{sec}$ \\
\hline$\lambda_{S M S, \text { attack }}$ & $495 \mathrm{msgs} / \mathrm{city} / \mathrm{sec}$ \\
& $9 \mathrm{msgs} / \mathrm{sector} / \mathrm{sec}$ \\
\hline$\lambda_{S M S, \text { regular }}$ & $138.6 \mathrm{~K} / \mathrm{city} / \mathrm{hr}$ \\
& $0.7 \mathrm{msgs} / \mathrm{sector} / \mathrm{sec}$ \\
\hline
\end{tabular}

of a vulnerability), reconnaissance (characterization of the conditions necessary to attack the vulnerability), exploit (attacking the vulnerability) and recovery (cleanup and forensics). We approach targeted SMS attacks in the same fashion. Enck, et al. [12] provide a methodology for executing such an attack; we summarize it here.

The vulnerability in GSM cellular networks that allows for targeted text message attacks to occur is the result of bandwidth allocation on the air interface. Under normal operating conditions, the small ratio of bandwidth allocated to control versus traffic data is sufficient to deliver all messages with a low probability of blocking. However, because text messages use the same control channels as voice calls for delivery (SDCCHs), contention for resources occurs when SMS traffic is elevated. Given a sufficient number of SMS messages, each of which require on average four seconds for delivery [27], arriving voice calls will be blocked for lack of available resources.

Sending text messages to every possible phone number is not an effective means of attacking a network. The haphazard submission of messages is in fact more likely to overwhelm gateways between the Internet and telecommunications networks than to disrupt cellular service. An adversary must efficiently blanket only the targeted area with messages so as to reduce the probability of less effective collateral damage. The information to achieve such a goal, however, is readily available. Using tools including NPA-NXX Area Code Databases, Internet search engines and even feedback from service provider websites, an attacker can easily construct a "hitlist" of potential targets. Armed with this information, an adversary can then begin exploiting the bandwidth vulnerability.

The exploit itself involves saturating sectors to their SDCCH capacity for some period of time. In so doing, the majority of attempts to establish voice calls are blocked. For all of Manhattan, which would typically be provisioned with 12 SDCCHs per sector, a perfectly executed attack would require the injection of only 165 messages per second, or approximately 3 messages/sector/second. Because downtime in telecommunications networks has historically proven expensive [10], we more fully characterize these attacks such that effective solutions can be developed.

\subsection{Attack Characterization}

In order to judge the efficacy of any countermeasure against tar- 


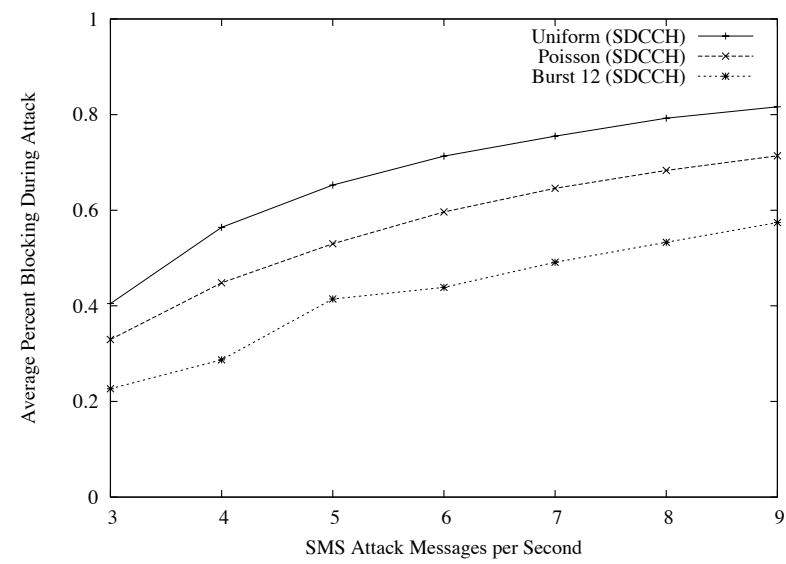

Figure 3: The blocking probability for traffic exhibiting deterministically uniform, Poisson and bursty arrival patterns over varying attack strengths.

geted SMS attacks, it is necessary to fully characterize such an event. We seek to understand the observed conditions and the subtle interplay of network components given a wide range of inputs. To achieve these ends, we have developed a detailed GSM simulator. The design considerations and verification of its accuracy are discussed in the Appendix.

A cellular deployment similar to that found in Manhattan [27] is used as our baseline scenario. The variables and parameters of this scenario are summarized in Tables 1 and 2. Each of the 55 sectors in the city has $12 \mathrm{SDCCHs}^{1}$. We assume both call requests and text messages arrive with a Poisson distribution and that $\mathrm{TCH}$ and SDCCH holding times are exponentially distributed around the appropriate means unless explicitly stated otherwise. Such values are well within standard operating conditions [19, 25, 21].

To isolate the impact of blocking caused by SDCCH congestion, we do not include pre-SDCCH queues; we examine the impact of such queues in Sections 4 and 5. If a call request of text message arrives when all SDCCHs are occupied, the request is blocked.

A sector is observed for a total of 60 minutes, in which the middle 30 minutes are exposed to a targeted SMS attack. The SMS attack intensity is varied between 4 and 13 times the normal SMS load, i.e., $\lambda_{S M S}=165 \mathrm{msgs} / \mathrm{sec}$ (3 messages/second/sector) to $\lambda_{S M S}=495 \mathrm{msgs} / \mathrm{sec}(9 \text { messages } / \text { second } / \text { sector })^{2}$. All results are the average of 1000 runs, each using randomly generated traffic patterns consistent with the above parameters.

We first replicate the attack presented in Enck et al [12]. To do this, a burst of 12 SMS messages is sent in sequential frames once every four seconds to each sector. The SDCCH holding time for each message is made deterministic and set to exactly $4 \mathrm{sec}$ onds. This bursty behavior quickly loads all SDCCHs. Whereas telecommunications networks are traditionally designed to experience blocking probabilities of less than $1 \%[29,30,5]$, this attack is able to prevent more than $90 \%$ of all calls from being completed due to SDCCH blocking.

Because delay variability is likely throughout the network, and because SDCCH holding times will not be deterministic due to varying processing times and errors on the wireless links, this per-

\footnotetext{
${ }^{1}$ In reality, only the highest capacity sectors would be so overprovisioned [27], making this a conservative estimate for every sector in a city.

${ }^{2}$ Because DoS attacks on the Internet frequently exhibit more than an entire year's volume of traffic [31], such an increase is relatively insignificant.
}

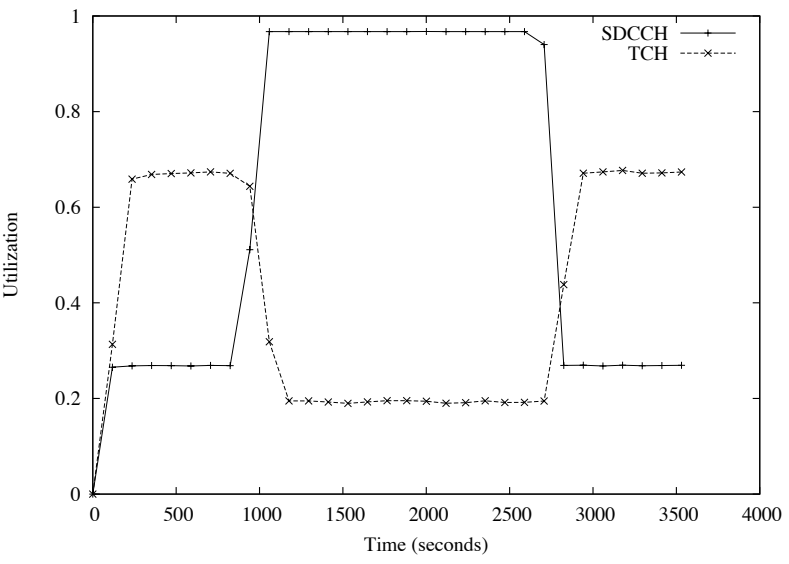

Figure 4: The utilization of SDCCHs and TCHs for an attack exhibiting a Poisson interarrival at a rate of 495 messages/second.

fect attack would be difficult to achieve in real networks. Accordingly, we investigate a number of flow arrival characteristics while considering exponentially distributed SDCCH holding times. Figure 3 illustrates the effectiveness of attacks when messages arrive with a Poisson, bursty or deterministically uniform distribution. Notice that, due to the addition of variability, bursty attacks are the least successful of the three. This is because it is unlikely that 12 text messages arriving back-to-back will all find unoccupied SDCCHs. Thus blocking occurs on the attack messages, and legitimate traffic that arrives between bursts has a higher probability of finding an available SDCCH. The most effective attack is when messages arrive uniformly spaced; however, due to variable network delay, such an attack would also be difficult to realize.

Our remaining experiments therefore assume a Poisson distribution for the arrival of text messages. We use an attack intensity of $495 \mathrm{msgs} / \mathrm{sec}$, which is equal to 9 message/second/sector and yields a blocking probability of $71 \%$. For this case we show the SDCCH and TCH utilization in Figure 4. This figure confirms the effectiveness of the attack: during the attack, the SDCCH utilization is near 1.0, and the TCH utilization drops from close to $70 \%$ down to approximately $20 \%$. This shows that although TCHs are available for voice calls, they cannot be allocated due to SDCCH congestion. Our experiments confirm that, at this rate, no other bottlenecks in the system exist, including other control channels or the SS7 signaling links.

\section{MITIGATION TECHNIQUES}

Voice communications have traditionally received priority in telecommunications systems. Because voice has been the dominant means by which people interact via these networks, providers allow for the degradation of other services in order to achieve high availability for the voice services. There are, however, an increasing set of scenarios in which the priority of services begins to change.

On September 11th, 2001, service providers experienced significant surges in usage. Verizon Wireless reported the number of calls made increased by more than $100 \%$ above average levels. Cingular Wireless experienced an increase of over $1000 \%$ for calls bound for the greater Washington D.C area [27]. In spite of the increased call volume, SMS messages were still received in even the most inundated areas because the control channels used for their delivery remained uncongested. In both emergency and day-to-day situations, the utility of text messaging has increased to the same level as voice communications for significant portions of the population [2]. 
For this reason, attractive mitigation solutions must not only protect voice services from targeted SMS attacks, but also allow SMS service to continue. In particular, differentiated service for text message delivery based upon the source of the SMS traffic is desirable. For instance, authenticated messages originated by emergency responders should be given higher priority than messages submitted by unauthenticated sources.

There are three traditional approaches to combating congestion. The first is rate limitation of the traffic source - in this case the interfaces on which messages are submitted. The network can also be protected by shedding traffic or using scheduling mechanisms. Finally, resources may be reallocated to alleviate the network bottleneck. We examine these solutions below.

\subsection{Current Solutions}

Cellular providers have introduced a number of mitigation solutions into phone networks to combat the SMS-based DoS attacks. These solutions focus on rate limiting the source of the messages and are ineffective against all but the least sophisticated adversary. To illustrate, the primary countermeasure discovered by the authors of the original study was a per-source volume restriction at the SMS gateway [12]. Such restrictions would, for example, allow only 50 messages from a single IP address. The ability to spoof IP addresses and the existence of zombie networks render this solution impotent. Another popular deployed solution filters SMS traffic based on the textual content. Similar to SPAM filtering, this approach is effective in eliminating undesirable traffic only if the content is predictable. However, an adversary can bypass this countermeasure by generating legitimate looking SMS traffic from randomly generated simple texts, e.g. "I will meet you at Trader Joe's at 5:00pm. -Alice"

Note that these and the overwhelming majority of other solutions deployed in response to the SMS vulnerability can be classified as edge solutions. Ineffective by construction, such solutions try to regulate the traffic flowing from the Internet into the provider network at its edge. Provider networks cover huge geographic areas and consist of hundreds of thousands of network elements. Any compromised element can be a conduit for malicious traffic. Moreover, if left unregulated, the connections between provider networks can also be exploited to inject SMS traffic.

Rate limitation is largely unattractive even within the core network. The distributed nature of Short Messaging Service Centers (SMSCs), through which all text messages flow, makes it difficult to coordinate real-time filtering in response to targeted attacks.

Therefore, for the purposes of this discussion, we assume that an adversary is able to successfully submit a large number of text messages into a cellular network. The defenses below are dedicated to protecting the resource that is being exploited in the SMS attack - the bandwidth constrained SDCCHs. Note that the Internet faces a similar conundrum: once dominant perimeter defenses are failing in the face of dissolving network borders, e.g., as caused by wireless connectivity and larger and more geographically distributed networks [18]. As is true in the Internet, we must look to other methods to protect telecommunications networks.

In the following sections we discuss mitigation techniques based on queue management and resource provisioning. For each solution we provide some basic analysis to provide insight; the motivation for parameter selection is covered in more detail in Section 5 .

\subsection{Queue Management Techniques}

\subsubsection{Weighted Fair Queueing}

Because we cannot rely on rate limitation at the source of mes- sages, we now explore network-based solutions. Fair Queueing [26] is a scheduling algorithm that separates flows into individual queues and then apportions bandwidth equally between them. Designed to emulate bit-wise interleaving, Fair Queueing services queues in a round-robin fashion. Packets are transmitted when their calculated interleaved finishing time is the shortest. Building priority into such a system is a simple task of assigning weights to flows. Known as Weighted Fair Queueing (WFQ) [9], this technique can be used to give incoming voice calls priority over SMS.

We provide a simplified analysis to characterize the performance of WFQ in this scenario. We apply WFQ to the service queues of the SDDCH. We create two waiting queues, one for voice requests and one for SMS requests, respectively. The size of the call queue is 6 and the size of the SMS queue is 12 (discussed in Section 5). To determine the relative blocking probability and utilization of the voice and SMS flows, we begin by assuming the conditions set forth in Tables 1 and 2.

WFQ can be approximated as a general processor sharing system (GPS) [35]. The average service rate of such systems is the weighted average of the service rates of all classes of service requests. In our case we have two types of requests: voice calls with $\lambda_{\text {call }}=0.2525$ calls $/$ sector $/$ sec and an average service time on the SDCCH of $\mu_{\text {call }}^{-1}=1.5$ seconds, and SMS requests with $\lambda_{S M S}=9.7 \mathrm{msgs} / \mathrm{sector} / \mathrm{sec}$ (attack traffic + regular traffic) and $\mu_{S M S}^{-1}=4$ seconds. Therefore, for our system, $\mu^{-1}=3.94$ seconds.

Although our system has multiple servers (SDCCHs), and is thus an $\mathrm{M} / \mathrm{M} / \mathrm{n}$ system, because it is operating at high loads during an attack, it may be approximated by an $\mathrm{M} / \mathrm{M} / 1$ system with its $\mu=n \mu^{\prime}$, where $\mu^{\prime}$ is the service rate calculated above. Using these values, and accounting for the weighting of 2:1 for servicing call requests, the call traffic intensity $\lambda_{\text {call }} / \mu_{\text {call }}=\rho_{\text {call }}=0.04$, and the expected call queue occupancy is about $1 \%$. Because the $\rho_{S M S}$ is much greater than 1, its SMS queue occupancy is approximately $100 \%$. When combined, the total queue occupancy is approximately $67 \%$.

These numbers indicate that the WFQ-based approach would sufficiently protect voice calls from targeted SMS attacks. Section 5 offers additional insight through simulation.

\subsubsection{Weighted Random Early Detection}

Active queue management has received a great deal of attention as a congestion avoidance mechanism in the Internet. Random Early Detection (RED) [13, 7], one of the better known techniques from this field, is a particularly effective means of coping with potentially damaging quantities of text messages. While traditionally used to address TCP congestion, RED helps to prevent queue lockout and was therefore investigated. RED drops packets arriving to a queue with a probability that is a function of the weighted queue occupancy average, $Q_{a v g}$. Packets arriving to a queue capacity below a threshold, $t_{\text {min }}$, are never dropped. Packets arriving to a queue capacity above some value $t_{\max }$ are always dropped. Between $t_{\min }$ and $t_{\max }$, packets are dropped with a linearly increasing probability up to $P_{d r o p, \max }$. This probability, $P_{d r o p}$, is calculated as follows ${ }^{3}$ :

$$
P_{d r o p}=P_{d r o p, \max } \cdot\left(Q_{a v g}-t_{\min }\right) /\left(t_{\max }-t_{\min }\right)
$$

The advantages to this approach are twofold: first, lockout becomes more difficult as packets are purposefully dropped with greater frequency; secondly, because the capacity of busy queues stays

\footnotetext{
${ }^{3}$ Some variants of RED additionally incorporate a count variable. Equation 1 is the simplest version of RED defined by RFC 2309 [7].
} 
closer to a moving average and not capacity, space typically exists to accommodate sudden bursts of traffic. However, one of the chief difficulties with traditional RED is that it eliminates the ability of a provider to offer quality of service (QoS) guarantees because all traffic entering a queue is dropped with equal probability. Weighted Random Early Detection (WRED) solves this problem by basing the probability a given incoming message is dropped on an attribute such as its contents, source or destination. Arriving messages not meeting some priority are therefore subject to increased probability of drop. The dropping probability for each class of message is tuned by setting $t_{\text {priority, } \min }$ and $t_{\text {priority, } \max }$ for each class.

We consider the use of authentication as a means of creating messaging priority classes. For example, during a crisis, messages injected to a network from the Internet by an authenticated municipality or from emergency personnel could receive priority over all other text messages. A number of municipalities already use such systems for emergency [32] and traffic updates [36]. Messages from authenticated users within the network itself receive secondary priority. Unauthenticated messages originating from the Internet are delivered with the lowest priority. Such a system would allow the informative messages (i.e. evacuation plans, additional warnings, etc) to be quickly distributed amongst the population. The remaining messages would then be delivered at ratios corresponding to their priority level. We assume that packet priority marking occurs at the SMSCs such that additional computational burden is not placed on base stations.

Here, we illustrate how WRED can provide differentiated service to different classes of SMS traffic using the attack scenario described in Tables 1 and 2. We maintain separate queues, which are served in a round robin fashion, for voice requests and SMS requests. We apply WRED to the SMS queue. In this example we assume legitimate text messages arrive at a sector with an average rate of $0.7 \mathrm{msgs} / \mathrm{sec}$ with the following distribution: $10 \%$ high priority, $80 \%$ medium priority, and $10 \%$ low priority. The attack generates an additional $9 \mathrm{msgs} / \mathrm{sec}$.

To accommodate sudden bursts of high priority SMS traffic, we choose an SMS queue size of 12. Because we desire low latency delivery of high priority messages, we target an average queue occupancy $Q_{\text {avg }}=3$.

To meet this objective, we must set $t_{l o w, \min }$ and $t_{l o w, \max }$. For $\mathrm{M} / \mathrm{M} / \mathrm{n}$ systems with a finite queue of size $m$, the number of messages in the queue, $N_{Q}$, is:

$$
N_{Q}=P_{Q} \frac{\rho}{1-\rho}
$$

where:

$$
P_{Q}=\frac{p_{0}(m \rho)^{m}}{m !(1-\rho)}
$$

where:

$$
p_{0}=\left[\sum_{n=0}^{m-1} \frac{(m \rho)^{n}}{n !}+\frac{(m \rho)^{m}}{m !(1-\rho)}\right]^{-1}
$$

Setting $N_{Q}=3$, we derive a target load $\rho_{\text {target }}=0.855$. $\rho_{\text {target }}$ is the utilization desired at the SDCCHs. Thus, the packet dropping caused by WRED must reduce the actual utilization, $\rho_{\text {actual }}$ or $\lambda_{S M S} /\left(\mu_{S M S} \cdot n\right)$, caused by the heavy offered load during an attack, to $\rho_{\text {target }}$. Therefore:

$$
\rho_{\text {target }}=\rho_{\text {actual }}\left(1-P_{\text {drop }}\right)
$$

where $P_{\text {drop }}$ is the overall dropping probability of WRED. For traffic with average arrival rate of $\lambda_{S M S}=9.7 \mathrm{msgs} / \mathrm{sec}, \rho_{\text {actual }}=$ 3.23. Solving for $P_{d r o p}$,

$$
P_{\text {drop }}=1-\frac{\rho_{\text {target }}}{\rho_{\text {actual }}}=0.736
$$

$P_{d r o p}$ can be calculated from the dropping probabilities of the individual classes of messages by $\left(\lambda_{\text {low }}=9.07\right)$ :

$P_{\text {drop }}=\frac{P_{\text {drop }, \text { high }} \cdot \lambda_{\text {high }}+P_{\text {drop }, \text { med }} \cdot \lambda_{\text {med }}+P_{\text {drop }, \text { low }} \cdot \lambda_{\text {low }}}{\lambda_{S M S}}$

Because we desire to deliver all messages of high and medium priority, we set $P_{d r o p, \text { high }}=P_{d r o p, \text { med }}=0$. Using Equation 7, we find $P_{\text {drop }, \text { low }}=0.787$. This value is then used in conjunction with Equation 1 to determine $t_{\text {low,min }}$ and $t_{\text {low,max }}$.

The desired average queue occupancy, $Q_{a v g}$, is 3 . From equation $1, t_{\text {low, min }}$ must be an integer less than the average queue occupancy. This leaves three possible values for $t_{\text {low,min }}: 0,1$, and 2 . The best fit is found when $t_{\text {low, min }}=0$ and $t_{l o w, \max }=4$, resulting in $75 \%$ dropping of low priority traffic.

Using this method it is possible to set thresholds to meet delivery targets. Of course, depending on the intensity of an attack, it may not be possible to meet desired targets according to Equation 7, i.e., it may not be possible to limit blocking to only low priority traffic. While the method outlined here provides just an approximate solution, given the quantization error in setting $t_{l o w, \min }$ and $t_{l o w, \max }$ (they must be integers), we believe the method is sufficient. We provide more insight into the performance of WRED in Section 5.

\subsection{Resource Provisioning}

None of the above methods deal with the system bottleneck directly; rather, they strive to affect traffic before it reaches the air interface. An alternative strategy of addressing targeted SMS attacks instead focuses on the reallocation of the available messaging bandwidth. We therefore investigate a variety of techniques that modify the way in which the air interface is used.

To analyze these techniques we resort to simple Erlang-B queueing analysis. We present a brief background here. For more details see Schwartz [35]. In a system with $n$ servers, and an offered load in Erlangs of $A$, the probability that an arriving request is blocked because all servers are occupied is given by:

$$
P_{B}=\frac{\frac{A^{n}}{n !}}{\sum_{l=0}^{l=n-1} \frac{A l}{l !}}
$$

The load in Erlangs is the same as the utilization, $\rho$, in a queueing system; it is simply the offered load multiplied by the service time of the resource. The expected occupancy of the servers is given by:

$$
E(n)=\rho\left(1-P_{B}\right)
$$

\subsubsection{Strict Resource Provisioning}

Under normal conditions, the resources for service setup and delivery are over-provisioned. At a rate of 50, 000 calls/hour in our baseline scenario, for example, the calculated average utilization of SDCCHs per sector is approximately $2 \%$. Given this observation, if a subset of the total SDCCHs can be used only by voice calls, blocking due to targeted SMS attacks can be significantly mitigated. Our first air interface provisioning technique, Strict Resource Provisoning (SRP), attempts to address this contention by allowing text messages to occupy only a subset of the total number of SDCCHs in a sector. Requests for incoming voice calls can compete for the entire set of SDCCHs, including the subset used for SMS. In order to determine appropriate parameters for systems using SRP, we apply Equations 8 and 9. 


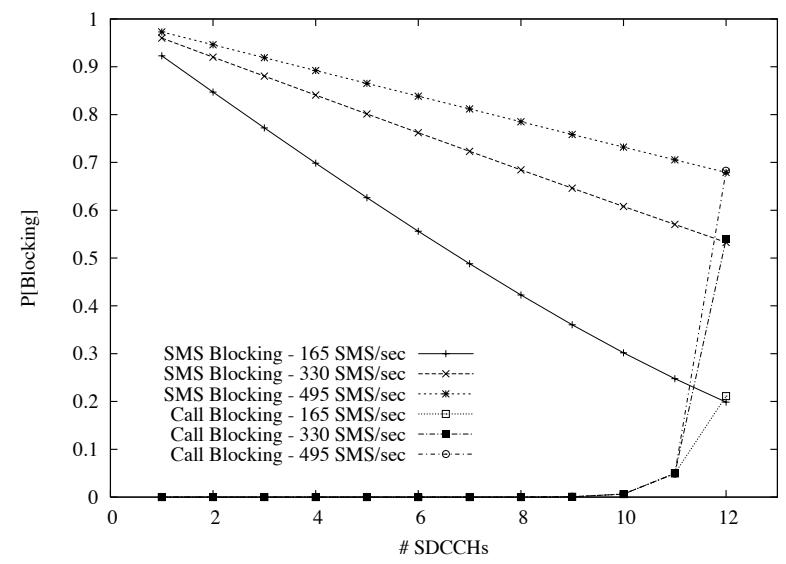

Figure 5: The probability that incoming calls and SMS messages are blocked in a system implementing SRP. We vary the number of SDCCHs that will accept SMS requests from 1 to 12(all).

To isolate the effectiveness of SRP, we consider a system with no queue. Figure 5 shows the blocking probabilities for a system using SRP when we vary the number of SDCCHs that will accept SMS requests from 1 to 12 (all). Because incoming text messages only compete with voice calls for a subset of the resources, any resulting call blocking is strictly a function of the size of the subset of voice-only SDCCHs. The attacks of intensity 165, 330, and 495 msgs/sec (3, 6, and 9 messages/second/sector) have virtually no impact on voice calls until the full complement of SDCCHs are made available to all traffic. In fact, it is not until 10 SDCCHs are made available to SMS traffic that the blocking probability for incoming voice calls reaches $1 \%$.

By limiting the number of SDCCHs that will serve SMS requests, the blocking for SMS is increased. When only six SDCCHs are available to text messages, blocking probabilities for SMS are as high as $84 \%$. Because significant numbers of people rely upon text messaging as their primary means of communication, such parameters should be carefully tuned. We will discuss the impact of additional factors after examining the results of simulation in Section 5 .

\subsubsection{Dynamic Resource Provisioning}

While SRP reprovisions capacity on existing SDCCHs, other over-provisioned resources in the sector could be manipulated to alleviate SDCCH congestion. For example, at a rate of 50,000 calls/hour, each sector uses an average of $67 \%$ of its TCHs. If a small number of unused TCHs could be repurposed as SDCCHs, additional bandwidth could be provided to mitigate such attacks.

Our second air interface technique, Dynamic Resource Provisioning, attempts to mitigate targeted text messaging attacks by temporarily reclaiming a number of TCHs (up to some limit) for use as SDCCHs. This approach is highly practical for a number of reasons. First, increasing the bandwidth (762 bits/second) of individual SDCCHs is difficult without making significant changes to the either the radio encoding or the architecture of the air interface itself. Because major changes to the network are extremely expensive and typically occur over the course of many years, such fixes are not appropriate in the short term. Secondly, dynamically reclaiming channels allows the network to adjust itself to current conditions. During busy hours such as morning and evening commutes, for example, channels temporarily used as SDCCHs can be returned to the pool of TCHs to accommodate elevated voice traffic

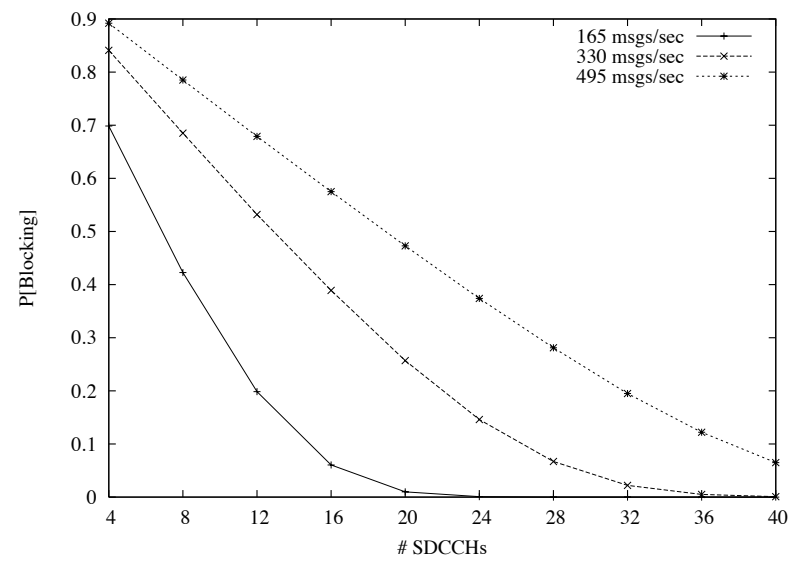

Figure 6: The probability of an incoming call/message blocking in a sector for a varying number of SDCCHs

needs. Lastly, because SDCCHs are assigned via the AGCH, allocating incoming requests to seemingly random timeslots requires almost no changes to handset software.

Figure 6 demonstrates the blocking probability for incoming calls and text messages in a sector using DRP to add a variable number of SDCCHs. Again, no queue was used. The ability of an attacker to block all channels is significantly reduced as the number of SD$\mathrm{CCHs}$ increases. Attackers are therefore forced to increase the intensity of their attack in order to maintain its potency. For attacks at a rate of $165 \mathrm{msgs} / \mathrm{sec}$, doubling the number of available SD$\mathrm{CCHs}$ reduces the calculated blocking caused by an attack by two orders of magnitude. The blocking probability caused by attacks at higher rates, in which the number of Erlangs is greater than the number of SDCCHs, decreases in roughly a linear relationship to the number of SDCCHs added.

One potential drawback with DRP is that by subtracting TCHs from the system, it is possible to increase call blocking because of TCH exhaustion. In fact, the reclamation of TCHs for use as SDCCHs increases the blocking probability for voice calls from $0.2 \%$ in the base scenario (45 TCHs, $12 \mathrm{SDCCHs}$ ) to $1.5 \%$ where $40 \mathrm{SDCCHs}$ are available (a reduction to $40 \mathrm{TCHs}$ ). Section 5 offers additional insight into the tradeoffs inherent to this scheme.

\subsubsection{Direct Channel Allocation}

The ideal means of eliminating the competition for resources between call setup and SMS delivery would be through the separation of shared mechanisms. Specifically, delivering text messages and incoming call requests over mutually exclusive sets of channels would prevent these flows from interfering with each other. The challenge of implementing such a mechanism is to do so without requiring significant restructuring of the network architecture. As previously mentioned, such fundamental changes in network operation are typically too expensive and time consuming to be considered in the short term. While the SRP technique provides a rudimentary separation, it is possible to further isolate these two types of traffic.

As mentioned in the previous section, DRP is easily implementable because the AGCH specifies the location of the SDCCH allocated for a specific session. After call requests finish using their assigned $\mathrm{SDCCH}$, they are instructed to listen to a specific TCH. Because the use of a TCH is the eventual goal of incoming voice calls, it is therefore possible to shortcut the use of SDCCHs for call setup. Incoming calls could therefore be directed to a TCH, leaving SDC$\mathrm{CHs}$ exclusively for the delivery of SMS messages. This technique, 


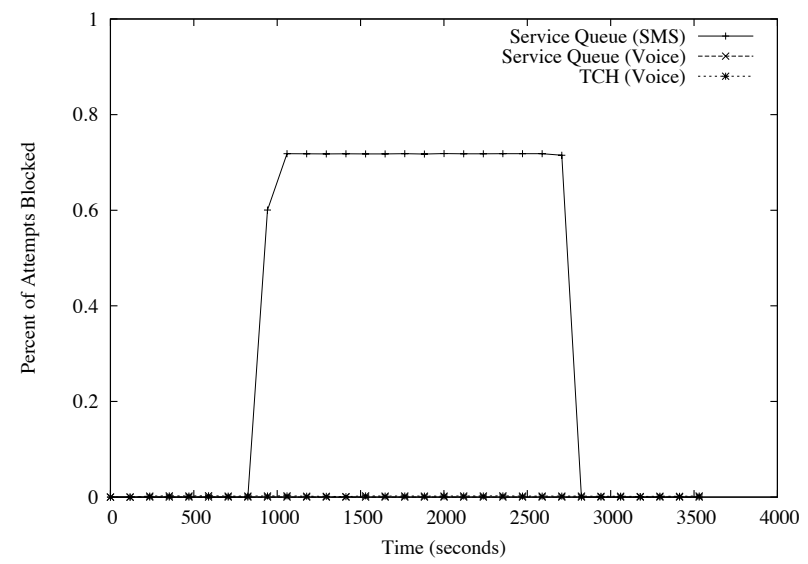

Figure 7: The simulated blocking probability for a sector implementing WFQ. Notice that voice calls are unaffected by the attack, whereas the majority of text messages are dropped.

which we refer to as Direct Channel Allocation (DCA), removes the shared SDCCH channels as the system bottleneck.

Calculating blocking probabilities for a system implementing DCA is a simple matter of analyzing SDCCH and TCH blocking for the two independent flows. For $165 \mathrm{msgs} / \mathrm{sec}$, text messages have a calculated blocking probability of approximately $20 \%$. This value increases to $68 \%$ as the attack intensity increases to $495 \mathrm{msgs} / \mathrm{sec}$. Voice calls, at an average rate of 50, 000 calls $/$ hour, have a blocking probability of $0.2 \%$. Note that because the shared bottleneck has been removed, it becomes extremely difficult for targeted text messaging attacks to have any effect on voice communications. In Section 5, we will highlight these new potential points of contention.

\section{RESULTS AND DISCUSSION}

In order to characterize each of our proposed mitigation techniques, we simulate attacks against networks with the parameters of Tables 1 and 2 unless otherwise noted. The RACH parameters used optimal settings [20], and we recorded no blocking occurred due to RACH or SS7 signaling link overload during any simulations.

\subsection{Queue Management Strategies}

In the following two subsections we present our simulation results for WFQ and WRED. For both solutions, we maintain queues of size 6 and 12 for call requests and SMS messages, respectively. We experimented with several values of queue size and found these to provide a good balance between additional latency and robustness. Note that because the arrival rate of SMS messages is greater than the processing rate $(\rho>1)$, no finite queue can prevent dropping.

\subsubsection{Weighted Fair Queueing}

Buffering alone is not sufficient to protect against congestion [26, 17]. We apply WFQ with a weight of 2 for calls and 1 for SMS messages to ensure voice calls receive a suitable amount of SD$\mathrm{CCH}$ bandwidth.

Figure 7 illustrates the resulting blocking for a sector implementing WFQ. The preferential treatment of voice traffic eliminates the blocking previously seen in an unprotected system. Incoming text messages, however, continue to experience roughly the same blocking (72\%) observed by all traffic in the base attack scenario. As is shown in Figure 8, the queue itself does nothing to prevent conges-

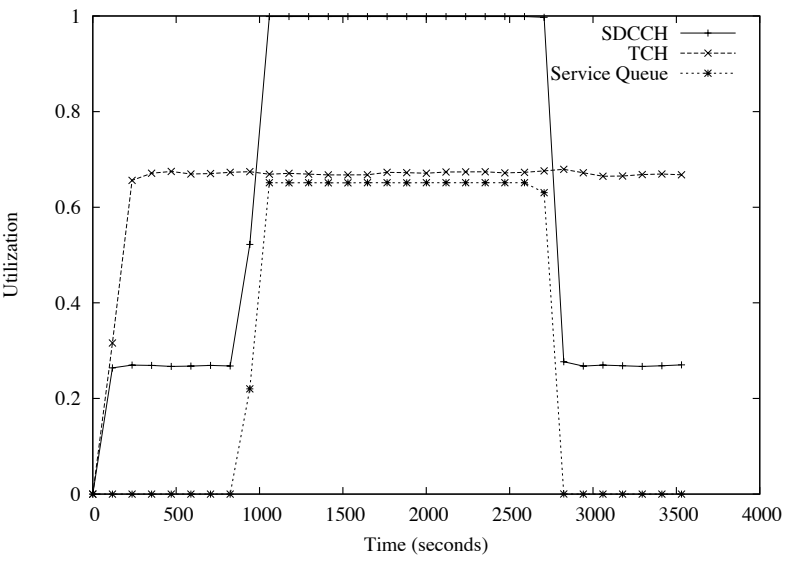

Figure 8: The simulated utilization for a sector implementing WFQ. Notice that TCH utilization remains constant throughout the attack.

tion. Total queue utilization is $65 \%$. As two-thirds of the queue space is available to text messaging, this represents a near total average occupancy of the SMS queue and a virtually unused voice traffic queue. Such an observation confirms our analytical results. This figure also demonstrates the ability to protect voice services, as TCH utilization is not lowered during the attack.

The advantage to implementing the WFQ mechanism is not only its relative simplicity, but also its effectiveness in preventing degradation of voice services during targeted SMS attacks. Unfortunately, the granularity for prioritizing text messages is insufficient to provide adequate service to those users relying upon text messaging as their dominant means of communication. While this can be done by adding one queue per SMS class, this solution will result in inefficient memory use and complexity. We discuss means of adding such granularity through the use of WRED.

\subsubsection{Weighted Random Early Detection}

The use of a prioritized dropping policy allows a system to offer similar prioritization to WFQ while maintaining only a single queue. In our implementation of WRED, we maintain one queue for voice requests (size of 6) and one queue for SMS messages (size 12) and apply WRED to the SMS queue. We differentiate the SMS traffic by setting different thresholds for each class. We assume that SMS traffic is marked upstream as having high, medium, or low priority. We assign the thresholds as $\left(t_{\text {high, } \max }=\right.$ $\left.t_{\text {high,min }}=12\right)$, medium $\left(t_{\text {med,max }}=10, t_{\text {med, } \text { min }}=6\right)$ and low $\left(t_{l o w, \max }=4, t_{\text {low, } \min }=0\right)$ priority. These priorities correspond directly to emergency priority users, network customers and Internet-originated messages, respectively. $Q_{a v g}$ is maintained as a simple weighted average with a weight of 0.8 on the most recent sample.

Figure 9 gives the blocking for each of the three priorities of text messages. Because voice calls never block in these simulations, we omit them from this graph. Both high and medium priority flows also do not experience blocking throughout the simulations. The blocking of Internet-originated messages averages $77 \%$, approximately the same blocking probability experienced by all incoming messages in the base attack scenarios. Service queue utilization, shown in Figure 10, is $20 \%$. With a total queue capacity of 18 , this corresponds to an average occupancy of 3.88 messages. Also notice that the TCH occupancy is maintained throughout the attack.

The parameters used in this simulation are the same as those in Section 4. We set the medium priority thresholds to allow some loss at very high loads to protect the high priority traffic under ex- 


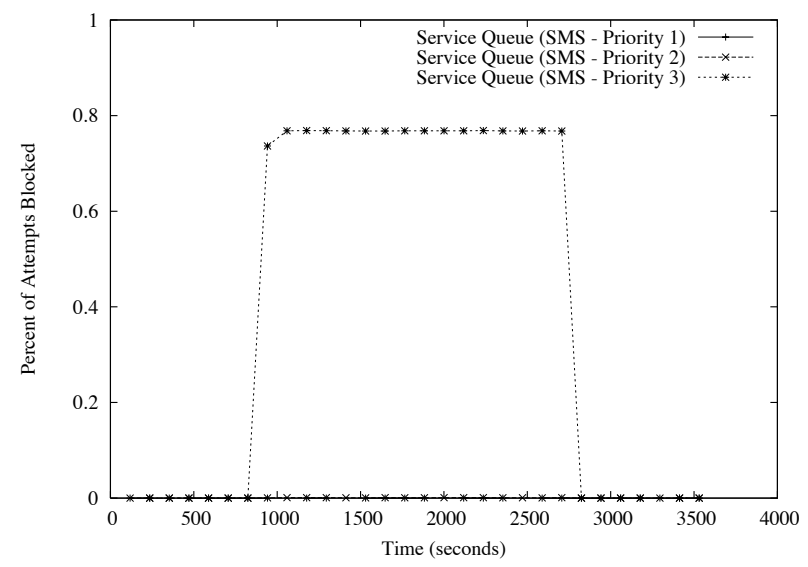

Figure 9: The simulated blocking probability for a sector implementing WRED. Unlike WFQ, only Internet-originated text messages are dropped at an elevated frequency.

treme circumstances, but because our average queue occupancy is about 3.9, no dropping of medium priority messages occurs. This matches well with our analytical results.

Systems implementing WRED not only match the elimination of voice call blocking seen through the use of WFQ, but also offer significantly improved performance in terms of message delivery. Implementing this solution, however, faces its own challenges. The authentication of high priority messages, for example, would require the use of additional infrastructure. High priority messages originating outside the network, such as emergency messages distributed by a city, may require the use of a dedicated line and/or the use of a public key infrastructure (PKI) for authentication. Because of historical difficulties effectively achieving the latter [11], implementing such a system may prove difficult. Even with such protections, this mechanism fails to protect the system against insider attacks. If the machine responsible for sending high priority messages into the network or user phones are compromised by malware, systems implementing WRED lose their messaging performance improvements over the WFQ solution. Note that networks not bounding priority to specific geographic regions can potentially be attacked through any compromised high priority device.

\subsection{Air Interface Strategies}

\subsubsection{Strict Resource Provisioning}

Before characterizing the SRP technique, careful consideration was given to the selection of operating parameters. Because many MSCs are capable of processing up to $500 \mathrm{~K}$ calls/hour, we engineer our solution to be robust to large spikes in traffic. We therefore allow SMS traffic to use 6 of the 12 total SDCCHs, which yields a blocking probability of $1 \%$ of voice calls by the SDCCH when voice traffic requests reach 250,000 calls/hour. (Note that calls would experience an average blocking probability of $71 \%$ due to a lack of TCHs with requests at this intensity.) Because these networks are designed to operate dependably during elevated traffic conditions, we believe that the above settings are realistic.

The blocking probabilities for SMS and voice flows in a sector implementing SRP are shown in Figure 11. Because SRP prevents text messages from competing for all possible SDCCHs, voice calls experience no blocking on the SDCCHs throughout the duration of the attack. Text messages, however, are blocked at a rate of $83 \%$. Channel utilization, illustrated in Figure 12, gives additional insight into network conditions. Because calling behavior remains the same during the attack, the resources allocated by the network

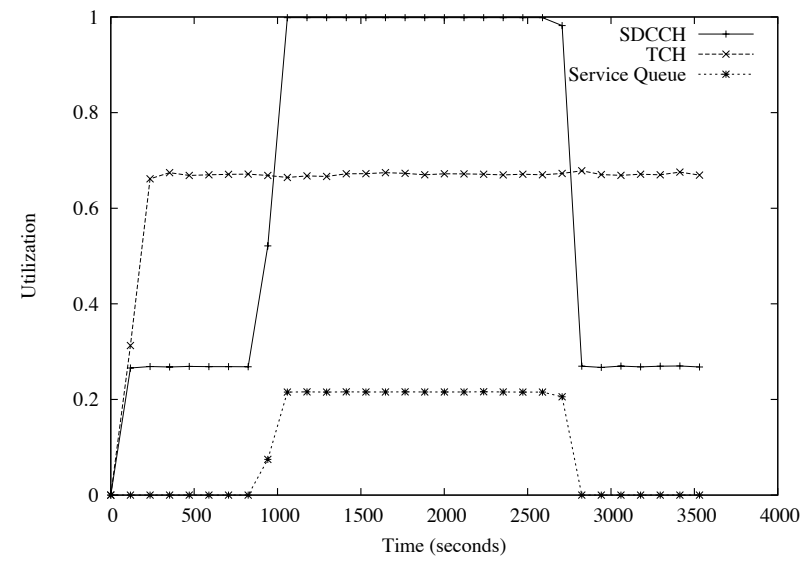

Figure 10: The simulated utilization for a sector implementing WRED. Notice that the queue occupancy stays low due to the decreased priority of Internet-originated messages.

are more than sufficient to provide voice service to users. By design, SDCCH utilization plateaus well below full capacity. While the SDCCHs used by text messages have an average utilization of $97 \%$, the SDCCHs used by incoming voice calls average a utilization of $6.3 \%$. This under-use of resources represents a potential loss of utility as the majority of text messages (legitimate or otherwise) go undelivered.

The difficulty with this solution is correct parameter setting. While theoretical results indicate that allocating 10 SDCCHs only increases call blocking to $1 \%$, voice traffic volumes fluctuate throughout the day. Provisioning resources in a static fashion must account for worst-case scenarios and therefore leads to conservative settings. While protecting the network from an attack, such a mechanism may actually hinder the efficiency of normal operation. When traffic channels are naturally saturated, as may be common during an emergency, such hard limits actually prevent users from communicating. Determining the correct balance between insulation from attacks and resource utilization becomes non-trivial. Accordingly, we look to our other techniques for more complete solutions.

Although it is possible to reclaim any number of TCHs for use as SDCCHs under the DRP mechanism, we limited the candidate number of channels for this conversion to two. In these experiments, a single $\mathrm{TCH}$ was repurposed into 8 SDCCHs every 10 minutes during the attack. This separation was designed to allow the network to return to steady state between channel allocations. While converting only two channels is not enough to completely eliminate attacks at high intensities, our goal is to understand the behavior of this mechanism.

The blocking probabilities for SMS and voice flows in a sector implementing the DRP technique are illustrated in Figure 13. As TCHs are converted for use as SDCCHs, the blocking probabilities for both incoming SMS and voice requests fall from $72 \%$ to $53 \%$ and eventually $35 \%$. This represents a total reduction of the blocking probability by approximately half. Call blocking due to $\mathrm{TCH}$ exhaustion was not observed despite the reduced number of available TCHs. Figure 14 illustrates a gradual return towards preattack TCH utilization levels as additional SDCCHs are allocated. The effects of the reprovisioning are also obvious for SDCCH utilization. The downward spikes represent the sudden influx of additional, temporarily unused channels. While SDCCH utilization quickly returns to nearly identical levels after each reallocation, more voice calls can be completed due to a decrease probability of the attack holding all SDCCHs at any given time. 


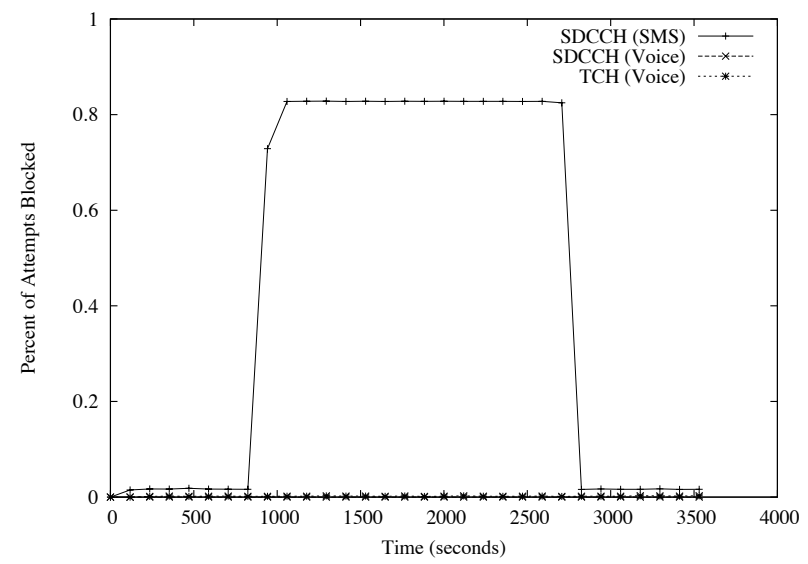

Figure 11: Blocking for a sector implementing SRP

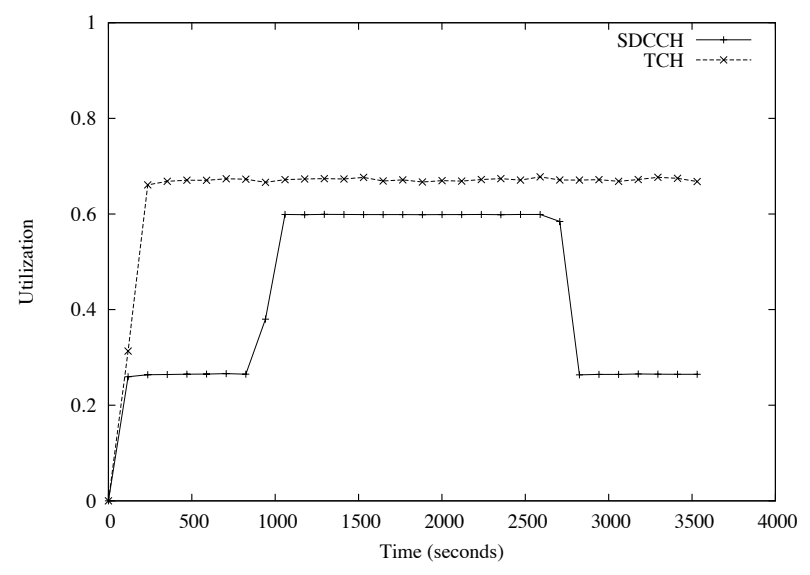

Figure 12: Channel utilization under SRP

As was a problem for SRP, determining the correct parameters for DRP is a difficult undertaking. The selection of two TCHs for conversion to SDCCHs illustrates the utility of this mechanism, but is not sufficient for real settings. To reduce the blocking probability on SDCCHs below the values observed for TCHs, a total of 48 SDCCHs would have to be made available. This leaves $39 \mathrm{TCHs}$, which results in a call blocking probability of $2.1 \%$ due to $\mathrm{TCH}$ exhaustion. Elevations in the volume of voice calls would likely require the release of some number of reclaimed TCHs to be repurposed to their original use.

The decision to convert channels is also non-trivial. Whereas the decision to reallocate channels at specific times was decided statically in our simulation, dynamically determining these parameters would prove significantly more challenging. Basing reclamation decisions on small observation windows, while offering greater responsiveness, may result in decreased resource use due to thrashing. If the observation window becomes too large, an attack may end before appropriate action can be taken. As was observed for SRP, the static allocation of additional SDCCHs faces similar inflexibility problems. Low resource utilization under normal operating conditions again represents a potential loss of opportunity and revenue.

\subsubsection{Direct Channel Allocation}

To simulate the DCA mechanism, incoming voice calls skip directly from the $\mathrm{RACH}$ to the next available TCH. An average of

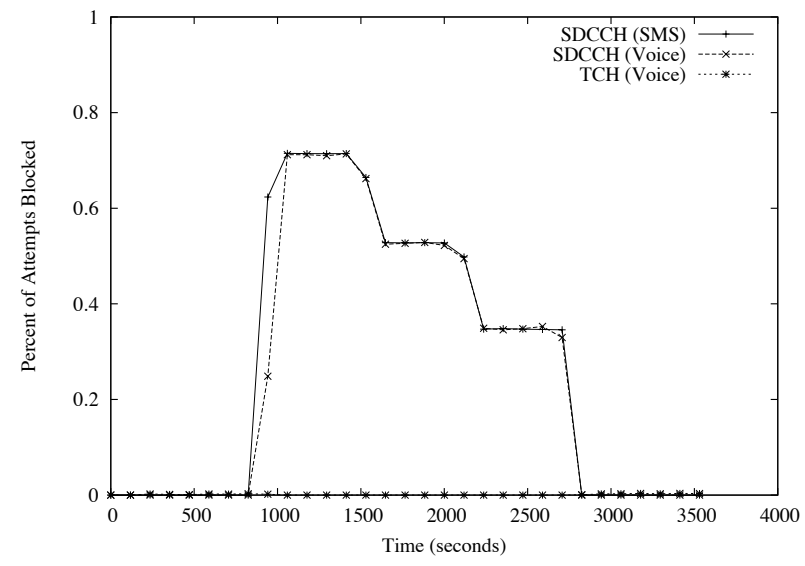

Figure 13: Blocking for a sector implementing DRP

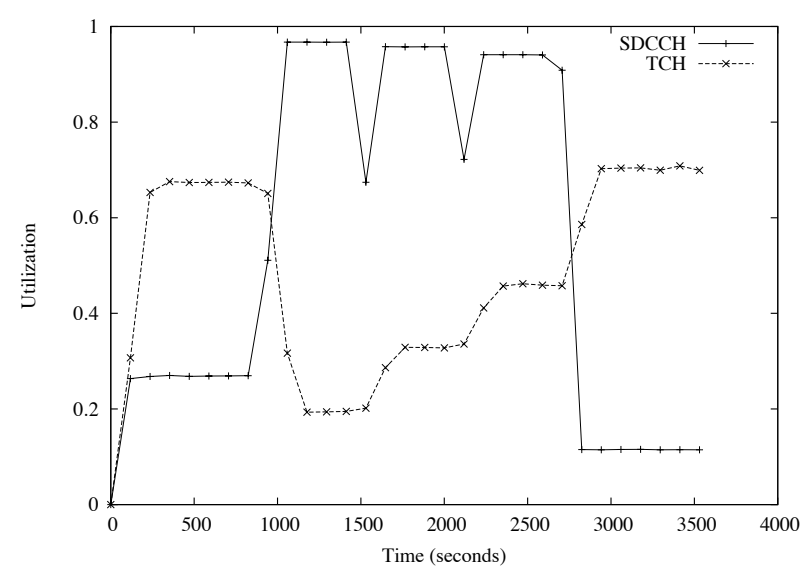

Figure 14: Channel utilization under DRP

1.5 additional seconds was added to each incoming call duration to account for the processing formerly occurring on an SDCCH. As is shown in Figure 15, voice calls arriving in a sector implementing the DCA scheme experience no additional blocking during a targeted SMS attack. Figure 16 confirms the results in the previous figure by showing the constant $\mathrm{TCH}$ utilization throughout the duration of the attack. No additional assistance is provided for the delivery of text messages under DCA.

While removing the bottleneck on the shared path of SMS delivery and voice call setup, DCA potentially introduces new vulnerabilities into the network. One advantage of using SDCCHs to perform call establishment is that users are authenticated before they are assigned TCHs. Under the DCA model, however, valuable traffic channels can be occupied before users are ever authenticated. Using a single phone planted in a targeted area, an attacker could simply respond to all paging messages and then ignore all future communications from the network. Because there are legitimate reasons to wait tens of seconds for a phone to reply to a page, an attacker could force the network to open and maintain state for multiple connections that would eventually go unused. Note that because paging for individual phones occurs over multiple sectors, a single rogue phone could quickly create a black-hole effect. Such an attack is very similar to the classic SYN attack observed throughout the Internet. While seemingly the most complete, the potential for additional damage made possible because of the DCA approach should be carefully considered. 


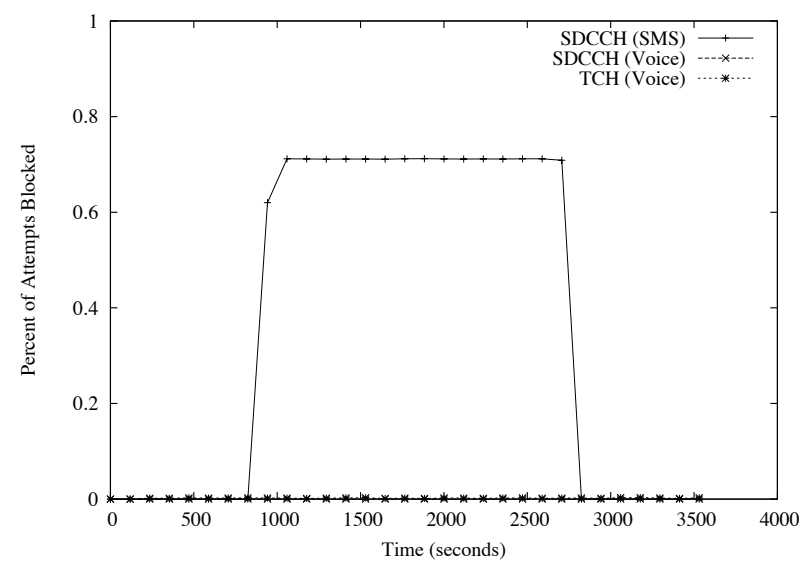

Figure 15: Blocking for a sector implementing DCA

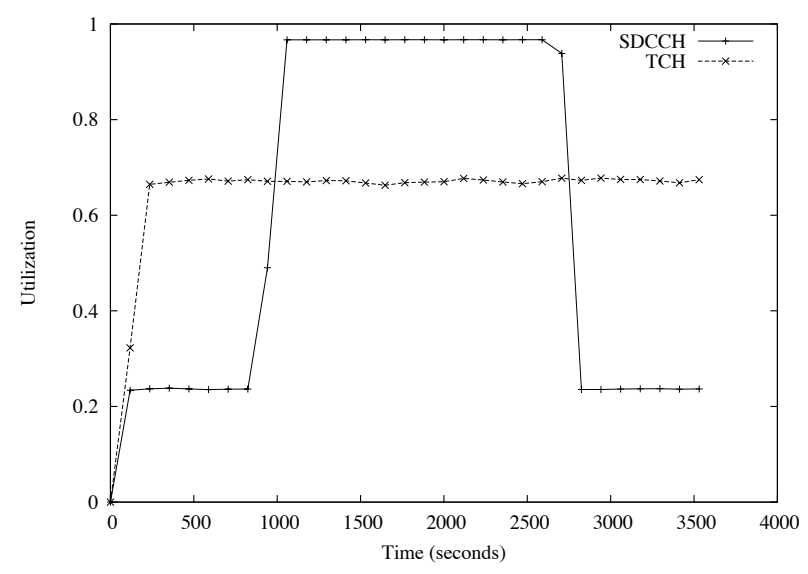

Figure 16: Channel utilization under DCA

\subsection{Combining Mechanisms}

There is no "silver-bullet" for maintaining a high quality of service for both text messaging and voice calls during a targeted SMS attack. As the above techniques demonstrate, each potential solution has its own weaknesses. The combination of such solutions, however, offers techniques robust to a wider array of threats. We examine two examples in which the fusion of mechanisms provides additional protections.

While directly addressing the bandwidth issue that makes targeted SMS attacks possible, the DRP technique lacks granularity to separate incoming voice and SMS requests. WRED, on the other hand, provides such traffic classification but is unable to react to attacks originating from trusted sources. To illustrate the benefits of layering these techniques, we increase the volume of legitimate traffic to $2 \mathrm{msgs} / \mathrm{sec}$, with $90 \%$ of that traffic being medium priority and the remaining $10 \%$ split equally between high and low priority flows. Such an increase would be representative of the elevated volumes of messages sent from crowded events such as concerts or public celebrations such as New Year's Eve gatherings. Figure 17 shows the result of the combination of the two techniques during an attack. Because of the naturally increased volume of legitimate traffic, subscriber-to-subscriber traffic experiences approximately $5 \%$ blocking in a sector only implementing WRED. As DRP activates and adds additional SDCCHs, only the attack traffic is dropped.

Another potentially beneficial combination is SRP and DRP. Given high volumes of voice traffic, a provider may not be able to repur-

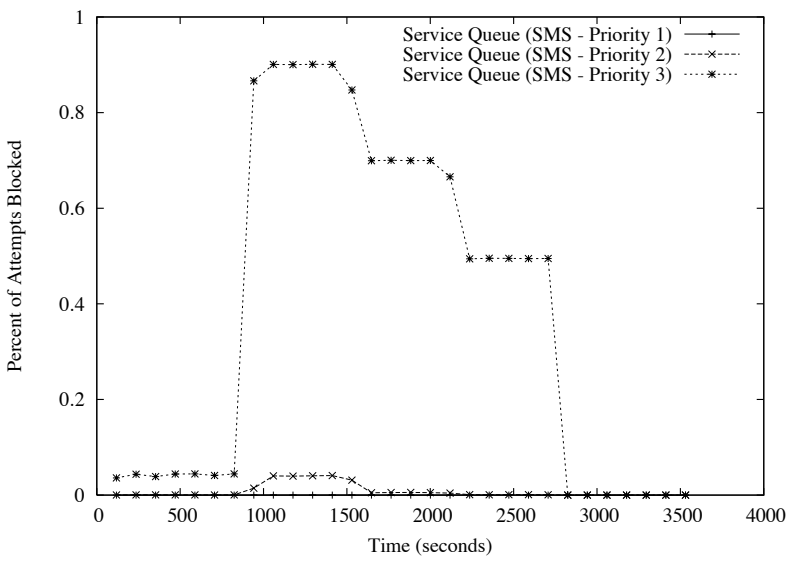

Figure 17: Blocking for a sector implementing both WRED and DRP.

pose enough SDCCHs to eliminate the effects of a targeted text messaging attack. Instead, a subset of the total channels could be reserved for voice requests. In so doing, voice blocking due to targeted text messaging attacks could be eliminated. All additional channels could be added to reduce blocking for text messages.

While no examples were uncovered during the course of this research, the combination of mitigation techniques should also be carefully considered. This fusion may lead to the creation of new or magnification of previously mentioned vulnerabilities. Accordingly, additional testing should be conducted before such integration could occur.

\section{CONCLUSION}

We have demonstrated the feasibility of targeted text messaging attacks and considered measures to combat them. Our analysis shows that adversaries with limited resources can cause call blocking probabilities to rise to as much as $70 \%$, effectively incapacitating a cellular network. Such vulnerabilities needn't be fatal. Our proposed countermeasures can mitigate or eliminate these attacks simply by changing the way in which call and SMS requests are handled.

The attacks discussed throughout are representative of growing and increasingly problematic class of vulnerabilities. The connectivity between the Internet and traditional voice networks introduces new avenues for exploit: once confined to exploiting only inert hosts, remote adversaries can debilitate the services we depend on to carry on our daily lives. In a broader sense, the ability to control the physical world via the Internet is inherently dangerous, and more so when the affected components are part of critical infrastructure. This work provides some preliminary solutions and analysis for these vulnerabilities. Essential future work will seek more general solutions that address these vulnerabilities in current and next generation networks.

\section{REFERENCES}

[1] The National Strategy to Secure Cyberspace. http: //www. us-cert. gov/reading_room/cyberspace_strategy • pdf, February 2003

[2] Young prefer texting to calls'. http://news.bbc.co.uk/2/hi/business/2985072.stm, June 2003 .

[3] 3rd Generation Partnership Project. Physical layer on the radio path; General description. Technical Report 3GPP TS 04.18 v8.26.0.

[4] 3rd Generation Partnership Project. Physical layer on the radio path; General description. Technical Report 3GPP TS 05.01 v8.9.0.

[5] A. Acampora and M. Naghshineh. Control and Quality-of-Service Provisioning in High- Speed Microcellular Networks. IEEE Personal Communications, 1(2):36-43, 1994. 
[6] S. Berinato. Online Extortion - How a Bookmaker and a Whiz Kid Took On an Extortionist and Won. CSO Online, May 2005.

[7] B. Branden, D. Clark, J. Crowcroft, B. Davie, S. Deering, D. Estrin, S. Floyd, V. Jacobson, G. Minshall, C. Partridge, L. Peterson, K. Ramakrishnan, S. Shenker, J. Wroclawski, and L. Zhang. RFC 2309 - Recommendations on Queue Management and congestion Avoidance in the Internet. rfc2309.txt, 1998.

[8] S. Byers, A. Rubin, and D. Kormann. Defending Against an Internet-based Attack on the Physical World. ACM Transactions on Internet Technology (TOIT), 4(3):239-254, August 2004

[9] A. Demers, S. Keshav, and S. Shenker. Analysis and Simulation of a Fair Queueing Algorithm. In Proceedings of ACM SIGCOMM, pages 3-12, 1989.

[10] L. Dryburgh and J. Hewett. Signaling System No. 7: The Role of SS7. http: //www.ciscopress.com/articles/article.asp?p= $330805 \backslash \& r l=1,2004$

[11] C. M. Ellison and B. Schneier. Ten Risks of PKI: What You're Not Being Told About Public-Key Infrastructure. Computer Security Journal, 16(1):1-7, 1999.

[12] W. Enck, P. Traynor, T. F. La Porta, and P. McDaniel. Exploiting Open Functionality in SMS-Capable Cellular Networks. In Proceedings of the ACM Conference on Computer and Communication Security (CCS), November 2005 .

[13] S. Floyd and V. Jacobson. Random Early Detection Gateways for Congestion Avoidance. IEEE/ACM Transactions on Networking, 1(4):397-413, August 1993

[14] C. Haney. NAI is latest DoS victim. http://security. itworld.com/ 4339/NWW116617_02-05-2001/page_1.html, February 52001

[15] J. Hedden. Math::Random::MT::Auto - Auto-seeded Mersenne Twister PRNGs. http: //search.cpan.org/ jdhedden/ Math-Random-MT-Auto-5.01/1 ib/Math/Random/MT/Auto.pm. Version 5.01.

[16] J. Ioannidis and S. Bellovin. Implementing Pushback: Router-Based Defense Against DDoS Attacks. In Proceedings of Network and Distributed System Security Symposium (NDSS), February 2002.

[17] R. Jain. Myths about congestion management in high speed networks. Internetworking: Research and Experience, 3:101-113, 1992.

[18] G. Kunene. Perimeter Security Ain't What It Used to Be, Experts Say. DevX.com, 2004.

[19] Lucent Technologies. 5ESS(R) 2000 - Switch Mobile Switching Centre (MSC) for Service Providers.

http: //www. lucent.com/products/solution/0, , CTID+ 2019-STID+10048-SOID+824-LOCL+1,00.html, 2006.

[20] C. Luders and R. Haferbeck. The Performance of the GSM Random Access Procedure. In Vehicular Technology Conference, pages 1165-1169, June 1994.

[21] K. Maney. Surge in text messaging makes cell operators :-). July 272005.

[22] Mike Grenville. Operators: Celebration Messages Overload SMS Network. http:

/ / www . 160 characters . org /news . php? action=view\&nid=819, November 2003

[23] J. Mirkovic and P. Reiher. A Taxonomy of DDoS Attacks and DDoS Defense Mechanisms. ACM SIGCOMM Computer Communication Review, 34(2):39-53, 2004

[24] D. Moore, V. Paxson, S. Savage, C. Shannon, S. Staniford, and N. Weaver. Inside the Slammer Worm. IEEE Security and Privacy, 1(4), July 2003.

[25] Motorola Corporation. Motorola GSM Solutions. www . motorola.com/ networkoperators/pdfs/GSM-Solutions . pdf, 2006.

[26] J. B. Nagle. On Packet Switches with Infinite Storage. IEEE Transactions on Communications, COM-35(4), April 1987.

[27] National Communications System. SMS over SS7. Technical Report Technical Information Bulletin 03-2 (NCS TIB 03-2), December 2003.

[28] Nyquetek, Inc. Wireless Priority Service for National Security. http: //wireless.fcc.gov/releases/da051650Publicuse.pdf, 2002.

[29] R. Ramjee, R. Nagarajan, and D. F. Towsley. On optimal call admission control in cellular networks. In Proceedings of the IEEE Conference on Computer Communications (INFOCOM), pages 43-50, 1996.

[30] R. F. Rey, editor. Engineering and Operations in the Bell System. Bell Telephone Laboratories, INC, second edition, 1984.

[31] M. Richtel. Yahoo Attributes a Lengthy Service Failure to an Attack. The New York Times, February 82000.

[32] Roam Secure. 17 Counties \& Cities in Washington, DC Region deploy Roam Secure Alert Network.

http: //www. roamsecure.net/story.php?news id=52, September 2005.

[33] P. Roberts. Al-Jazeera Sites Hit With Denial-of-Service Attacks. PCWorld Magazine, March 262003.

[34] S. Savage, D. Wetherall, A. Karlin, and T. Anderson. Practical network support for IP traceback. In Proceedings of ACM SIGCOMM, pages 295-306, October 2000.

[35] M. Schwartz. . In Telecommunication Networks - Protocols, Modeling and

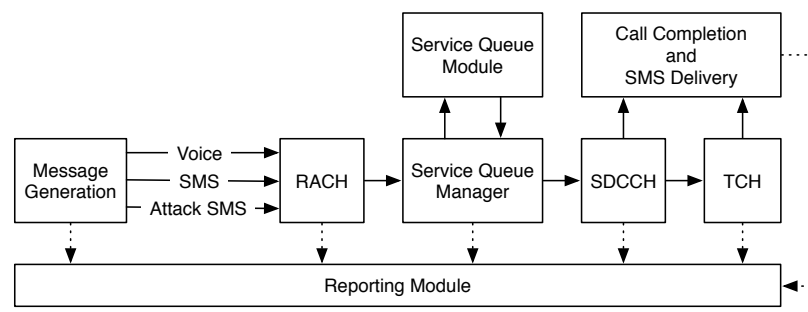

Figure 18: Simulator Architecture

Analysis. Addison-Wesley Publishing Company, 1987.

[36] Tamara Neale. VDOT LAUNCHES NEW 511 EMAIL ALERT SERVICE. http: //www.virginiadot.org/infoservice/news/ newsrelease. asp? ID=CO-511-06, February 2006

[37] B. Waters, A. Juels, J. Halderman, and E. Felten. New client puzzle outsourcing techniques for DoS resistance. In Proceedings of ACM CCS'04, pages 246-256, 2004.

\section{APPENDIX}

While analytics easily characterize simplistic network loads, it is difficult to analyze multi-faceted input flows. To more fully characterize targeted SMS attacks and mitigation techniques, we developed an extensible, detailed GSM simulator. A number of simulation environments, including OPNET and NS2, were considered. The GSM air interface modules for both OPNET and NS2 focus on mobility management and did not contain support for SMS. More importantly, neither design was amenable to modeling potential countermeasures. Commercial GSM simulators were also considered, but were highly complex, specialized, and cost prohibitive.

The core simulator is over 5,000 lines of $\mathrm{C}$ with supplement scripts written in various languages. In total, the project contains over 8,000 lines of code. All code was written with flexibility of configuration and extensibility in mind. Figure 18 depicts our simulator architecture. Solid lines indicate voice and SMS message flow; dashed lines indicate reporting of events, including creation, stage entrance, blocking, and completion. Simulation begins in the Message Generation stage, where messages are randomly created using a Mersenne Twister Pseudo Random Number Generator [15]. Message generation can follow Poisson, uniform, or bursty arrival patterns. After creation, messages proceed to the RACH stage, which strictly follows 3GPP TS 04.18 [3] and is tunable using the max_retrans and tx integer variables. The Service Queue Manager stage assigns messages to an SDCCH. If desired, a pluggable Service Queue Module can be defined using standard interface callback functions. If possible, the Service Queue Manager assigns a message to an SDCCH. Rather than simulating exact communication and compensating for retransmission, messages are held in the SDCCH stage for an exponential mean service time corresponding to message type. For accuracy, each SDCCH services messages by decreasing counters only during frames defined in 3GPP TS 05.01 [4]. When counters reach zero, SMS messages complete and voice messages attempt to acquire a $\mathrm{TCH}$. Like the SDCCH stage, the TCH stage uses an exponential mean hold time to simulate channel occupancy. TCHs service messages during every frame, and when the hold time counter reaches zero, the call is complete, and the $\mathrm{TCH}$ is released.

The accuracy of the simulator was verified by running simple base scenarios and comparing blocking and utilization to values obtained using equations 8 and 9 . Base scenarios similar to those in the paper were created: 12 SDCCHs, $45 \mathrm{TCHs}$, voice and SMS service times with exponential means of 1.5 and 4.0 seconds respectively, voice holding time with exponential mean of 120 seconds, voice call loads representing $25 \mathrm{~K}, 50 \mathrm{~K}, 75 \mathrm{~K}$, and $100 \mathrm{~K}$ calls per hour to Manhattan, and SMS messages loads of 1, 2, and 3 messages per second to a sector. Voice and SMS loads were simulated separately and the average blocking and utilization of 1,000 runs was compiled. Both blocking and utilization are measured on a scale from 0 to 1.0. Statistical analysis showed that $95 \%$ of all samples fell within 0.006 of the mean, with many close to 0.001 . For example, SDCCH utilization for the SMS load of $3 \mathrm{msgs} / \mathrm{sec}$ was $0.809609 \pm 0.00133944$. Compared to calculated blocking and utilization, all simulated means deviated less than $20 \%$, with all utilization means less than $2 \%$. All but one simulated blocking mean was within $10 \%$ calculated values. The outlier was resulted from simulating a very small blocking value and was off by $-5 \times 10^{-4}$, which therefore is acceptable. 